\title{
DIRECCIONES DE LA SEMIÓTICA EN COLOMBIA
}

\author{
Armando Silva * \\ Universidad Nacional de Colombia
}

Es interesante reconocer el renovado interés de la semiótica en Colombia al igual que en varios países de América Latina. Se puede decir que de la moda de los años setenta, donde muchos aficionados en Colombia se ocuparon de la semiología, amándola con la misma incomprensión y emoción furtiva con que hoy la reniegan, pasamos a otro momento profesional en el cual la dirección semiótica se constituye no sólo en una materia de estudio dentro de distintas disciplinas o carreras, sino en un verdadero problema teórico y paradigma epistemológico de las ciencias sociales, sobre el cual hay que reflexionar $y$, diría, no sólo pretender.

No obstante sigue siendo cierto que no podemos hablar de un gran avance de estos estudios en nuestro país, pero sí encontramos otra manera de encauzar sus análisis de modo más competente, con menos esperanzas inauditas y con mucho más ajuste a lo que de ella se pueda

* El autor de este artículo agradece al profesor Fabio Jurado de la Universidad Nacional de Colombia sus varios aportes en la actualización de la información que contiene este escrito. 
esperar. Al respecto, se podrían enumerar dos motivos que han impulsado su interés nacional. En 1987 se funda la Asociación Colombiana de Semiótica (ACSE) que agrupa a sus estudiosos a nivel nacional y cuya junta directiva se rota por ciudades, según la importancia que tenga en cada una de ellas, brindando así estímulo a su desarrollo regional. ACSE ha organizado simposios nacionales y ha generado algunas publicaciones que se enumerarán más adelante. De otro lado, cabe agregar que la evolución misma de la semiótica, a nivel de la disciplina misma, ha brindado otras posibilidades que encuadran mejor con las aspiraciones culturales de varios de nuestros científicos sociales, que ahora pueden ver en este campo de estudios un instrumento de ayuda metodológica y conceptual, además de principios necesarios de formalización, dentro de los objetos propios a su materia de investigación.

Una manera de registrar y dimensionar sus direcciones e intereses es consultar lo que se hace en las universidades donde existe como materia, lo cual también nos muestra ciertas tendencias por escuelas de pensamiento - incluso hasta por ciudades donde se estudia - debido a que se concentran ciertos docentes o investigadores con preocupaciones afines. De acuerdo con lo anterior, podría presentar el siguiente panorama, que responde sólo a un primer intento por sistematizar esta práctica profesional en nuestro país. Propongo seguir las mismas subdivisiones ya previstas en un ensayo que publiqué sobre la «Semiótica en América Latina» (Silva, 1993: 269-286). Hago el recorrido según una división tripartita que atiende sus usos disciplinarios escuetos de la siguiente manera: lo relacionado con los estudios discursivos, con los espacios o bien con las interacciones sociales. $O$ sea me refiero a una semiótica dicursiva, otra visual y la última socio-cultural. En el interior de esta clasificación se explicará lo relativo a centros de estudio o a tendencias un tanto más particulares de algunos docentes o investigadores. $\mathrm{Al}$ final presento un anexo sobre publicaciones y lugares de estudio.

\section{DISCURSOS Y ANÁLISIS TEXTUALES}

- Estudios Narratológicos. Precisamente vale la pena destacar al respecto el «Segundo Congreso Latinoamericano de Semiótica», al cual tuve la oportunidad de asistir en representación de Colombia, que tuvo lugar en Rosario, Argentina en 1987, donde fue claro el dominio y las preferencias de los colegas del continente por esta semiótica narrativa, con relevante demostración de interés por la obra de A.J. Greimas. 
De la misma manera esta dirección corresponde a una de las de mayor interés en Colombia, si tenemos en cuenta que en ella participan varios centros. Es el caso de la Universidad del Valle en Cali, donde existe un seminario permanente, dirigido por Eduardo Serrano (por ejemplo, sus análisis de la obra de García Márquez: Serrano, 1991), adscrito al Departamento de Letras y que toma como centro de reflexión la obra de A.J. Greimas y G. Genette, en cuanto análisis discursivo de la obra literaria.

En la Universidad Nacional de Bogotá, en el Departamento de Lingüística, desde 1986 se abrió, de la misma manera, el Seminario de semiótica a cargo de Juan Gómez con la participación de un grupo de ex alumnos con quienes se creó el Grupo de «Investigación Narratológica» de la Universidad Nacional, el cual ha conseguido sacar una revista Narratológicas (véase apéndice para información de ésta y otras publicaciones periódicas en Colombia), dedicada a un tema especializado en cada publicación, como la Focalización o el Relato. De donde han salido estudiantes con sinceros intereses investigativos, dentro de los cuales cito a Felipe Ardila, interesado en semiótica literaria, y a Rodrigo Argüello (1998) con interés por la semiótica del relato fílmico y urbano. A este grupo se unió Fabio Jurado, que, después de permanecer varios años en México, se vinculó a la Universidad Nacional en 1990 como profesor de semiótica en el Departamento de Literatura, adelanta varios trabajos de aplicación de isotópicas y los modelos actanciales sobre todo referidos a la obra de Juan Rulfo (1989). En esta línea trabajan jóvenes ex alumnos ya con algunas publicaciones de artículos en semiótica y literatura como son Luis Noriega y Miguel Manrique.

En las universidades Tecnológica de Tunja, con Benigno Ávila y Guillermo Bustamante, y la de Nariño, en la ciudad de Pasto, se consolida, de la misma manera, una tendencia hacia la semiótica literaria. Los objetos de estudios corresponden preferencialmente a análisis de obras literarias latinoamericanas y los enfoques de estudio se refieren a problemas de focalización, procesos de narración en la novelística, relaciones narrador-lector, hermenéutica textual y análisis isotópicos.

- Lingüistica Textual. Precisamente con este título el profesor Jaime Bernal publicó una interesante antología (Bernal, 1986), en la cual recogió ensayos de estudiosos colombianos, además de otros investigadores extranjeros, poniendo en evidencia los presupuestos y las direcciones de trabajo de esta tendencia en el país. En esta dirección de lingüística textual se orienta el seminario de semiótica del 
Instituto Caro y Cuervo, a cargo de Alfonso Ramírez (1986), con publicaciones al respecto y quien junto con el linguiística Felipe Pardo fundó hace unos años el Círculo Linguiístico de Bogotá.

- Teoría del signo. En esta dirección más emparentada con la filosofía que las anteriores, se trabaja, dentro del Departamento de Filosofía y Letras de la Universidad Nacional de Bogotá, sobre todo a través de las investigaciones del profesor Alfonso Rincón, especialista en semiótica medieval y con tesis doctoral sobre San Agustín (1992). También el tema es de interés de la seccional de Medellín en el Departamento de Humanidades, con la orientación de Juan Gonzalo Moreno hasta 1990 y del psicoanalista Hermes Pradilla a partir de entonces. Este Departamento organiza desde 1990, el Posgrado en Semiótica y Hermenéutica del Arte y ha incluido el área de semiótica narrativa y visual, lo cual viene estimulando nuevos objetos de análisis histórico, con aplicaciones de la teoría del signo a la moda, la fotografía, la publicidad y la ciudad, entre otros temas. Desde 1998 se ha creado la Maestría en Estudios Estéticos en la misma Universidad Nacional de Medellín, la cual ha tenido interesantes repercusiones en otras ciudades por su alta capacidad convocatoria al proponer seminarios sobre temas atractivos al público y a los académicos, como ciudad y ecología o ciudad y desechos. Sobre tales tópicos pueden citarse trabajos de ex alumnos que ya han publicado interesantes ensayos como es el caso de Jorge Echavarría, profesor de la Pontificia Bolivariana, sobre análisis iconográficos, de Raúl Domínguez sobre publicidad e historiografía o de Tarzicio Valencia en poesía.

En la Universidad Javeriana de Bogotá, se han interesado, igualmente, por los estudios filosóficos y hermenéuticos y sus cruces con la semiótica. Allí, adscritos a los departamentos de Filosofía y Comunicación Social, están vinculados los profesores Jaime Rubio, con varias publicaciones sobre P. Ricoeur (Rubio, 1987) y que ha liderado un seminario de reflexión permanente sobre semiótica y hermenéutica, y Mariluz Restrepo, que terminó su maestría en filosofía con una tesis (1989) sobre la obra completa de Peirce, llamando así de nuevo la atención sobre este fundador contemporáneo de la semiótica y presentando una investigación rigurosa y muy bien documentada.

- Pragmática y teorías de la enunciación. En este sentido se presentan dos enfoques que recogen diversas propuestas con énfasis en el 
aspecto pragmático de la comunicación. Podríamos decir que el interés en este aspecto crece y se encuentra muy ligado a estudios literarios y teatrales. Bajo una dimensión pragmática se orienta el posgrado en Lingüística de la Universidad del Valle, en la ciudad de Cali, que fue dirigido por Luis Baena. Dentro de los mismos intereses trabajan otros docentes del Departamento de Letras de la misma Universidad, como son Carlos Vásquez, con varios ensayos sobre literatura y teatro y Carlos Restrepo, coordinador de un nuevo proyecto de Maestría en Literatura con énfasis en análisis semióticos.

En la Universidad de los Andes, en Bogotá, pueden considerarse como próximas a esta tendencia algunas publicaciones de Monserrat Ordóñez aplicadas a textos literarios como la Vorágine de E. Rivera. En la Universidad Industrial de Santander también se perfila en esta dirección Serafín Martínez, con su trabajo literario aplicando análisis textuales y enunciativos a la novelística del santandereano recientemente fallecido Pedro Gómez. En la Universidad Nacional en Medellín, debo citar escritos de Jairo Montoya, con doctorado en la materia de la Universidad de Puerto Rico, para quien «si entre las palabras y los objetos se teje es un complejo campo de fuerzas y de entrecruzamientos la posibilidad de una semio-pragmática del discurso queda al mismo fundamentada» (1992: 55).

- Estudios literarios. La profesora Ángela Betancur lidera el grupo de estudios semióticos en el área de literatura, de la Facultad de Comunicaciones, de la Universidad de Antioquia. Este grupo aborda el análisis del texto literario a partir de la semiótica narrativa de Greimas y Fontanille, con algunos apoyos en la teoría narratológica de Genette. El libro Estudio semiótico de «En la diestra de Dios padre», cuyos autores son Ángela Betancur y José Decio Londoño, constituye un referente bibliográfico en los horizontes investigativos del grupo. Diversos artículos publicados en la revista Lingüística y Literatura, de la Universidad de Antioquia, sustentan el trabajo del grupo, con una influencia académica en la carrera de Linguística y en la Maestría en Literatura Colombiana de esta universidad.

- Semiótica y Pedagogía. Durante la década del 90 en Colombia se consolidaron grupos que venían reflexionando en torno a los procesos de aprendizaje de la lectura y la escritura. Entre estos grupos se destacan, por el enfoque semiótico que orientaron sus investigaciones, el liderado por Gloria Rincón en la Universidad del Valle; el grupo 
liderado por Julio Escamilla en la Universidad del Atlántico; el grupo liderado por Blanca Bojacá en la Universidad Distrital y el grupo liderado por Fabio Jurado en la Universidad Nacional. Cada uno de estos grupos ha realizado publicaciones, entre las que sobresalen: la revista Enunciación, de la Universidad Distrital; los libros Los procesos de la lectura, Los procesos de la escritura y Entre la lectura y la escritura (compilaciones realizadas por Guillermo Bustamante y Fabio Jurado); Fundamentos semiolingüísticos de la actividad discursiva, de Julio Escamilla; Investigación, escritura y educación, de Fabio Jurado; Lenguaje y mundos posibles, de Gladiz Jaimes y María Elvira Rodríguez; El texto explicativo, de Blanca Bojacá y Raquel Pinilla; Etnoeducación: proyectos literatura y Embrujos de amor, de James Cortés. El efecto académico e investigador de estos grupos se encuentra en el libro que señala los lineamientos curriculares para abordar el área de lenguaje en los distintos grados y ciclos de la escolaridad, pues el Ministerio de Educación Nacional convocó a miembros de estos grupos para que definieran y sustentaran los enfoques más pertinentes para el aprendizaje escolar de la lectura y la escritura y en las reflexiones sobre el lenguaje y la literatura. Se puede considerar que el enfoque dominante que se acoge en este texto está sustentado desde las semióticas de Greimas, Barthes, Eco y Peirce.

\section{ANÁLISIS SOBRE IMÁGENES Y ESPACIOS}

- Espacio Urbano y la Ciudad. Tendencia de estudio en la que se toma como objeto específico la ciudad. En la Facultad de Artes de la Universidad Nacional en Bogotá, dentro del Departamento de Arquitectura, existe la materia de semiótica y distintas publicaciones especializadas como $A / U$ (Asuntos Urbanos) a cargo del arquitecto argentino Juan Carlos Pérgolis (1986), con trabajos sobre teoría arquitectónica; tal revista se preocupa por estudiar la ciudad bajo criterios semióticos. También la ciudad es motivo de estudios semióticos en la Universidad Nacional, con sede en Medellín, dentro de la misma Facultad de Arquitectura, como las investigaciones del catalán Jaime Xibillé (1990), que realizó su tesis doctoral en la Universidad de Barcelona sobre el arte en la ciudad posmoderna, y Darío Ruiz quien, sin interesarse específicamente por una orientación semiótica, trabaja sobre varios supuestos del lenguaje y la de-construcción urba- 
na. Uno de los principales aportes prácticos conseguidos por este grupo de Medellín ha sido llamar la atención sobre los barrios con profundo sentido popular (como el Manrique) que, con motivo de la instalación de las vías del Metro, ha sido lesionado en su arquitectura y modo de vida. Uno de los medios más utilizados para la divulgación de sus propuestas de los estudiosos de Medellín ha sido el video y la fotografía.

- Comunicación visual. Dentro de la Facultad citada, pero en el Departamento de Bellas Artes, se fundó, por parte del autor de este artículo, en 1978, el área de Comunicación Visual, con cuatro distintos niveles de estudio en semiótica, referidos al Arte y Diseño Gráfico. De la Universidad Nacional han salido publicaciones dedicadas al estudio de iconografía y diseño popular de nuestras ciudades (A. Silva, 1986).

- Figuración y Arte. En la búsqueda de alternativas distintas a la crítica de arte, aparece un nuevo interés por esta materia. Hoy ya está en funcionamiento la Maestría en Hermenéutica y Semiótica del Arte en la Universidad Nacional de Medellín, bajo la coordinación inicial de Juan Gonzalo Moreno, Jairo Montoya, Jaime Xibillé, Aníbal Córdoba y el historiador Antonio Restrepo, orientada en la perspectiva de la Estética Semiótica (J. Montoya, ed., 1987). Ésta es la primera escuela de posgrado que hace énfasis definido en los acercamientos al arte desde una teoría del símbolo, lo cual va a generar nuevos estudiosos del arte, que estén en condiciones de relevar a los tradicionales críticos de arte en Colombia, poseedores más bien de una abusiva retórica para producir efectos periodísticos y de mercado y donde brillan por su ausencia los análisis más juiciosos y profundos dentro de las nuevas ciencias del lenguaje y sus vínculos con las otras ciencias sociales. El desarrollo actual del interés en la semiótica en Colombia debe reconocer lo que sucede en Medellín con el posgrado mencionado; además de los «Encuentros Semiológicos», auspiciados desde hace ya algunos años por la Secretaría de Educación del Departamento, con relevante demostración de acogida académica y el «Círculo Lingüístico de Medellín» (Jaime Rojas, Víctor Villa, Manuel Morales, J. Mario Cardona, Ramón Vázquez, Amando Correa, Consuelo Restrepo, Rogelio Tobón y Omar Gómez en su junta directiva); así como las actividades de otros grupos muy activos en conferencias, publicacio- 
nes y traducciones: como las realizadas por Luis Alfonso Palau en el Departamento de Humanidades de la Universidad Nacional con Sede en Medellín.

Con un sentido un tanto más sociológico, pero utilizando argumentos semióticos puedo citar una investigación embrionaria sobre el teatro en Santander, al nor-oriente de Colombia, a cargo del profesor Álvaro Góngora (1991). A esta investigación se han unido algunos de los estudiantes del posgrado de Estudios Semiológicos de la Universidad Industrial de Santander, otro grupo de trabajo en semiótica en Colombia, que viene evolucionando hacia estudios sobre teatralidad en la vida cotidiana, además de la línea de investigación narratológica. Sobre el mismo tema del teatro, pero más como teoría, puesta en escena y dramaturgia, está la orientación de Consuelo Restrepo en la Universidad de Antioquia. También consignaría en esta tendencia figurativa los trabajos de Eduardo Domínguez, de la Facultad de Diseño de la Pontificia Bolivariana de Medellín, pues si bien su trabajo es de orden historiográfico (1990) su objeto inmediato son los periódicos colombianos de distintas épocas concebidos como imagen, o sea, cómo sus diseños y diagramas generan estructuras aislables de significación.

- Cine y análisis de Medios de Comunicación. Bajo una orientación semiótica se dan cursos de cine en la Javeriana de Bogotá, a cargo de los docentes Germán Muñoz, especialista en tecno-imágenes y sus sistemas expresivos (además de traductor de trabajos de Ch. Metz), Gilberto Bello, con mayor énfasis sociológico, y Fernando Vásquez. No obstante ha sido la Universidad del Valle, en la Facultad de Comunicación Social, fundada en la década de los 70 por el profesor Jesús Martín-Barbero, donde han tenido un mayor desarrollo los análisis de medios (M-Barbero, 1987). En las Universidad Tecnológicas de Pereira y Armenia, se han incrementado los estudios en medios y ya existen estudios de posgrado que enfatizan el problema de la televisión o el cine, dentro de programas de educación.

- Arte y semiótica. En el Departamento de Literatura, de la Universidad Nacional, la profesora checa, Jarmila Jandova adelantó la traducción, directa del checo, de una parte de la obra de Jan Mukarovski, en la que se destacan artículos relacionados con las teo- 
rías sobre el signo y sus convergencias en la estética y en los procesos interpretativos de los textos de la cultura: el cine, el folclore, la poesía, el teatro, etc. Dicha traducción se encuentra en prensa para el año 2000.

\section{ANÁLISIS SEMIÓTICOS BAJO LA INTERACCIÓN SOCIAL}

- Estudios dialectológicos. Aquí deben destacarse los estudios del Instituto Caro y Cuervo, de Bogotá, uno de los centros de mayor prestigio en filología clásica de la lengua castellana que acaba de ser destacado con el premio Príncipe de Asturias (1999). Cabe mencionar el extraordinario trabajo que concluyó en el Atlas Lingüístico y Etnográfico, dirigido por el profesor Luis Flórez y publicado en varios volúmenes por la Imprenta Patriótica del mismo Instituto, el cual ha servido de modelo en otros países para levantar mapas sobre usos locales del idioma y de las costumbres de cada región. Esta obra se propuso recoger material linguístico según áreas geográficas colombianas dejando abiertas las puertas para posteriores interpretaciones, como afirma el grupo de investigadores. Por supuesto que, como recuerda Rubén Arboleda (1987: 33), con el Atlas tenemos la posibilidad de abordar temas como tabúes y eufemismo, etimología popular, comparación del habla campesina, urbana y semiurbana y otros aspectos más.

- Estudios sociolingüísticos. En este sentido se vienen haciendo distintas publicaciones y estudios. Bajo los estudios actuales, el énfasis se coloca en los mismos hablantes como sujetos sociales de comunicación, quienes desprenden distintas características en el uso de la lengua. Es el caso de la tendencia predominante de la Universidad de Antioquia, en Medellín, con investigaciones sobre fenómenos musicales populares o de ciertos usos urbanos del lenguaje a cargo de los docentes Rito Llerena (1985) y Víctor Villa, o bien teoría lógica y sociosemiótica, como algunos estudios sobre semiótica del silencio de Rogelio Tobón (1989), obra que obtuvo el premio departamental de 1991, que adjudica el Departamento de Antioquia a una obra inédita de valor investigativo. También puede citarse a la francesa Helen 
Puliquen, de la Universidad Nacional de Bogotá, quien viene adelantando un trabajo literario basado en la sociocrítica, basándose en Goldmann y Bajtín.

- Estudios etnológicos. En este caso me refiero a trabajos sobre el uso del lenguaje en comunidades, en especial en los sectores indígenas. Habría que citar en especial a las Facultades de Antropología, de las Universidades Nacional y Andes de Bogotá, en una mezcla de opciones investigativas dentro de las cuales lo semiótico, a pesar de no ser siempre fundante, es tenido en cuenta por algunos investigadores. En los Andes debe destacarse el interés por problemas simbólicos de la profesora Elizabeth Reichel, quien se acerca así a una concepción semiótica en las culturas indígenas que analiza, especialmente en la amazonía colombiana. En la Universidad Nacional, con mayor disposición al trabajo textual, cabe mencionar los trabajos de Genoveva Iriarte (1991), orientados a tradición oral y desarrollados según criterios de indexicalidad prescriptiva y de performatividad narrativa.

En este aparte quisiera mencionar los últimos artículos del profesor Guillermo Páramo de la Universidad Nacional, quien dentro de una sólida formación en lógica y antropología intenta formalizar las bases de una lógica «paracosistente» que entregue los presupuestos para una lógica de lo simbólico. Sin duda, este esfuerzo formalizador (Páramo, 1989) para la antropología puede seguirse con interés para los problemas propios de las interacciones de los que se ocupa últimamente la semiótica.

- Estudios semiolingüísticos. En esta dirección se trata de comprender lo discursivo hacia los procesos sociales de producción, trabajando en un límite texto-extratexto y por tanto sus investigadores lo hacen, bien en nivel de modelos teóricos, o bien diseñando estrategias para trabajo propiamente de campo. Aquí es de anotar el grupo CADIS - Círculo de Análisis del Discurso-, fundado por Julio Escamilla (1987: 49-50) y adscrito a la Universidad del Atlántico en Barranquilla, ciudad caribeña situada al norte del país que cuenta con estudios en Filología e Idiomas. Este grupo tiene a su cargo adelantar estudios sobre el uso específico del habla costeña en la zona norte de Colombia y su orientación se ubica en el análisis socio-semiológico del discurso. 
- Estudios sobre procesos de comunicación social. Corresponde a una tendencia «culturalista» que tiene amplia difusión en varias escuelas de Comunicación Social, estimulada por la misma FELAFACS (Federación Latinoamericana de Facultades de Comunicación), dentro de la cual se plantea el estudio de los receptores de mensajes (ver Silva, 1988), tomando diversas posiciones conceptuales y teóricas. La semiótica encuentra allí plena justificación y así se han realizado reuniones donde se examinan los aportes que puedan encontrarse para los estudios de comunicación. En realidad se trata de un momento de encuentro de dos disciplinas que, de distintos modos, han llegado a formularse preguntas similares y que en el aspecto cultural coinciden con un nuevo interés en el análisis desde los sujetos sociales, enfrentando así, en el estudio de la comunicación, un proceso de producción a uno de interpretación, tanto a nivel de las estrategias del texto (estudio sobre narratarios), como de las prácticas sociales (estudio sobre y desde los destinatarios y receptores-destinatarios).

\section{ALGUNOS PROGRAMAS O PROYECTOS CONCRETOS DE POSGRADO EN COLOMBIA, DONDE EXISTE UN INTERÉS EN EL ANÁLISIS DESDE PERSPECTIVAS SEMIÓTICAS}

En la actualidad conocemos los siguientes programas de posgrado, en distintas universidades colombianas:

- Doctorado en Educación con énfasis en pensamiento visual, en la Universidad Nacional de Colombia, con sede en Bogotá, para el año 2000. Este proyecto ha sido concebido en especial por dos docentes con formación semiótica, Fabio Jurado y Armando Silva. Se abrirán tres líneas para el estudio de la relación educacion-comunicación: imagen-cognición, imagen-imaginarios e imagen y medios.

- Posgrado en Estudios Semiológicos de la Universidad Industrial de Santander, dentro de tres líneas de trabajo institucional: la Semiótica Narrativa, Literatura y Teatralidad. Su énfasis está previsto en la investigación. Lo funda el profesor Álvaro Góngora; lo dirige hoy la profesora Cecilia Ogeda. 
- Posgrado en Semiótica y Hermenéutica del Arte, del Depto. de Humanidades de la Universidad Nacional de Medellín, bajo la dirección inicial del profesor Hermes Padilla y la actual de Jairo Montoya. Este posgrado viene intensificando una formación filosófica y crítica de la semiótica y se ha interesado por varias temáticas, en especial las relacionadas con deconstruccionismo, Semiótica Visual y posmodernidad urbana.

- Maestría en Estudios Estéticos de la Universidad Nacional de Medellín. Se encuentra concentrado allí el grupo de Medellín: Jairo Montoya, Jaime Xibille, Gonzalo Moreno, Aníbal Córdoba, Antonio Restrepo y los españoles Manuel Delgado y Pere Salaber. Trabajan sobre el pensamiento estético contemporáneo.

- Programa de Maestría en Comunicación de la Universidad Javeriana, bajo la dirección del padre Gabriel Jaime Pérez. Pone énfasis en investigación cultural y la semiótica está prevista dentro de una de las áreas denominadas como enfoques básicos.

- Programa de Maestría en Comunicación Educativa de la Universidad Tecnológica de Pereira, a cargo de la profesora Amanda Castiblanco. Funciona desde hace tres años y la semiótica es una de las materias fundamentales dentro del área de metodología y materias teóricas.

- Proyecto de posgrado en Literatura de la Universidad del Valle, a cargo del profesor Carlos Restrepo, con alguna participación de la semiótica en aspectos metodológicos.

- En la Universidad Pedagógica y Tecnológica de Tunja el profesor Benigno Ávila ha impulsado una especialización en Literatura y Semiótica, de la cual han egresado aproximadamente 50 profesores, cuyas monografías se han orientado desde la semiótica, con mucho énfasis en las teorías narratológicas y en las perspectivas del pensamiento de Eco y Greimas.

- El Instituto Caro y Cuervo, en Bogotá, fundó una línea de trabajo en sociocrítica, dentro del programa de Maestría en Lingüística y Literatura. En esta línea se aborda el pensamiento de Bajtín y la propuesta de Edmund Cros para el análisis del texto literario; cabe destacar que en la obra de Cros ocupa un lugar importante la semiótica de Greimas. Entre quienes han profundizado en el análisis semiótico-textual se destacan las profesoras Neyla Pardo y Helene Pouliquen, así como el profe- 
sor Diógenes Fajardo, cuyos artículos publicados en la revista Thesaurus, muestran la relevancia de la mirada semiótica en análisis de obras como las de García Márquez y Germán Espinoza.

\section{ALGUNAS PUBLICACIONES PERIÓDICAS EN COLOMBIA QUE SE OCUPAN DE ESTUDIOS DESDE PERSPECTIVAS SEMIÓTICAS}

Sobre Semiótica. Boletín informativo de la Asociación Colombiana de Semiótica. Bogotá. Edita Fabio Jurado. Dirección: Ciudad Universitaria. Unidad Camilo Torres, Bloque C, Módulo 4, oficinas 601 y 604 de Bogotá.

Thesaurus. Revista de publicaciones especializadas en filología y lenguaje, del Instituto Caro y Cuervo de Bogotá. Dirección: Apartado Aéreo 51.502, Bogotá, Colombia.

Signo y Pensamiento. Revista de la Facultad de Comunicación Social de la Universidad Javeriana. Dirección: Cra 7 n. ${ }^{\circ}$ 43-82, Bogotá.

Texto y Contexto. Revista de la Universidad de los Andes de Bogotá. Dirección: Cra. 1 Este n. ${ }^{\circ} 18 .^{a}, 10$, Bogotá.

Narratológicas. Del grupo de investigaciones narratológicas del Seminario de Semiótica de la Universidad Nacional de Colombia, Bogotá. Dirección: A.A. 41.804, Bogotá.

A/U-Asuntos Urbanos, de la Facultad de Artes de la Universidad Nacional de Colombia. Dirección: Universidad Nacional, Bogotá.

Revista Universidad del Valle. Dirección: Universidad del Valle, Cali, Colombia.

Lingüística y Literatura. Revista del Departamento de Lingüística y Literatura de la Universidad de Antioquia. Dirección: Apartado Aéreo 1226, Medellín.

Contextos. Revista de la Facultad de Ciencias de la Educación de la Universidad de Medellín. Dirección: Apartado Aéreo 1983, Medellín.

Coloquios Lingüísticos. Revista del Círculo Lingüístico de Medellín. Dirección: Apartado Aéreo 56.068, Medellín, Colombia. 
Ciencias Humanas. Revista de la Universidad Nacional de Medellín. Dirección: Universidad Nacional de Medellín, Medellín.

Alegoría. De la Universidad de Tunja en donde se publican capítulos de monografías sobre semiótica de estudiantes de esa ciudad y avances de investigación de los profesores de la misma

Forma y Función. Del Departamento de Linguiística, de la Universidad Nacional. Ha publicado artículos en torno a la semiótica discursiva y los análisis textuales de poesía, relato literario y narrativas en lenguas indígenas, así como acercamientos semióticos a tópicos relacionados con la educación y la ciencia.

Literatura. Del Departamento del mismo nombre en la Universidad Nacional de Colombia, en Bogotá. Se publican allí artículos sobre análisis semiótico de textos literarios, como los artículos de los profesores Fabio Jurado y Diógenes Fajardo.

Enunciación. Esta revista tiene como énfasis la semiótica del discurso en relación con tópicos y problemas de la educación y de la pedagogía. La revista está financiada por la Universidad Distrital, de Bogotá, a través de su Facultad de Ciencias y Educación, con aportes de la línea de investigación «Mejoramiento de la calidad de la enseñanza de la lengua materna». En esta línea de investigación se inscribe el proyecto «Conocimiento del mundo, conocimiento lingüístico y entorno socio cultural en la adquisición de la lengua materna: una propuesta para la educación pre-escolar», y el proyecto «Análisis de textos escritos por niños en contexto de evaluación», financiados por Colciencias, entre 1997-1999. La revista está estructurada en 5 secciones: Ponencias, Avances de investigación, Artículos, Traducciones y Reseñas. La Dirección de la revista está a cargo de Gladiz Jaimes y María Elvira Rodríguez. La sección de traducciones constituye un aporte significativo de la revista, en tanto da cuenta de investigaciones que en semiótica y análisis del discurso se adelantan en Europa y en Norteamérica. Autores como Marcellessi y Charadeau, han sido traducidos en la revista por las profesoras Blanca Bojacá, Raquel Pinilla y María Elvira Rodríguez. La revista es semestral y recibe colaboraciones en el apartado aéreo 3062, de Bogotá.

Forma y Función. Publica trabajos en lingüística, sociolinguística, etnolingüística, análisis del discurso y semiótica; su ámbito está centrado en las ciencias del lenguaje. La revista está financiada por la Facultad de Ciencias Humanas y coordinada por el Departamento de Lingüística. En relación con la semiótica, la revista ha publicado ar- 
tículos sobre la interpretación, la escritura y la lectura, análisis semióticos de poesía y de relato literario, semiótica textual. Se han publicado traducciones de artículos sobre semiótica discursiva, de autores como Greimas. La revista es semestral y en la actualidad está dirigida por Ruth Pappenheim. Recibe colaboraciones en el correo electrónico: rppapenh@bacata.usc.unal.edu.co.

Literatura. Teoría, Historia, Crítica. Esta revista está orientada hacia los estudios literarios, ya sean historiográficos o analítico-textuales. En cada número se publica al menos un artículo sobre semiótica literaria; las tendencias de la semiótica literaria provienen de Greimas, Eco, Bajtín y Mukarovsky. La revista está financiada por la Facultad de Ciencias Humanas, de la Universidad Nacional de Colombia, y está coordinada por el Departamento de Literatura. Tiene como directora a la traductora de Mukarovsky, Jarmila Jandova. Se publica anualmente y pueden enviarse colaboraciones al correo: revdlite@bacata.usc.unal.ecu.co.

Lenguaje. Surgió en la década del 70 en la Universidad del Valle, Cali, como un medio para divulgar los trabajos de investigación en el área del lenguaje; si bien sus propósitos apuntaban hacia lo propiamente linguiístico, con el tiempo la revista fue incorporando análisis sobre argumentación y sobre la semiótica del discurso. A través de esta revista se divulgó paulatinamente el enfoque semántico comunicativo, cuyos fundamentos se sustentan en una teoría sobre la significación, entendida como dimensión semiótica de la cultura. Luis Ángel Baena lideró el enfoque y coordinó la revista en las décadas de mayor productividad como lo fueron las décadas del 80 y del 90.

Lingüística y Literatura. Ésta es otra revista que ha mantenido su regularidad en las ediciones. Está editada por el Departamento de Lingüística y Literatura, de la Universidad Antioquia, de Medellín. Semejante a la revista Lenguaje, ha combinado la publicación de artículos específicos sobre lingüística y artículos sobre semiótica narrativa, con análisis de textos literarios, latinoamericanos y colombianos, desde la perspectiva de Greimas, Genette, Bajtín y Eco, así como análisis semióticos sobre otros diversos significantes de la cultura, como la canción popular, el grafitti y el discurso publicitario. Hasta 1999 se habían editado 30 números. Las colaboraciones para esta revista se pueden enviar al apartado aéreo 1226, de Medellín.

Alegoría. Esta revista constituye un medio para la divulgación de los trabajos de investigación que adelantan los estudiantes y los profesores de la especialización en Literatura y Semiótica, de la Universidad 
Pedagógica y Tecnológica de Tunja, Boyacá. Los análisis semióticos del texto literario son abordados desde la perspectiva de Greimas, Barthes y Eco. La revista está dirigida por Benigno Ávila.

Polifonía. Se trata también de una revista que se ubica en los ámbitos de los estudios lingüísticos y literarios, considerando las aproximaciones semióticas a los textos como una franja de la misma, pues también aparecen artículos de crítica literaria y de profundización en análisis textual. La revista está adscrita al Departamento de Lenguas Modernas, de la Universidad del Atlántico, Barranquilla.

A las anteriores puede agregarse el «Banco de Información Semiótica» que adelanta el posgrado de Estudios Semiológicos de la Universidad Industrial de Santander y que ya ha ofrecido a las instituciones colombianas con intereses en el área. La biblioteca en semiótica de tal centro puede considerarse la de mayor registro en publicaciones periodicas extranjeras en Colombia. Dirección: Universidad Industrial de Santander. Centro de Estudios Semiológicos, profesor Álvaro Góngora, Director. Bucaramanga.

Proyecto Cultura Urbanas en América Latina y España desde sus imaginarios sociales. Dirigido por el profesor Armando Silva, prevé un enfoque cultural sobre la base de una metodología semiótica. Es el primer estudio que compara culturas urbanas de América desde una misma metodología y sobre presupuestos comunes. Recibe apoyo internacional de varias instituciones culturales, como el Convenio Andrés Bello del pacto Andino, la UNESCO, la ONU y distintas oficinas locales como la alcaldía de las ciudades de Buenos Aires y Bogotá o las universidades públicas como Universidad Autónoma de México y la Universidad de São Paulo en Brasil. Para el año 2001 saldrá una enciclopedia que reunirá los distintos trabajos por cada ciudad investigada.

\section{Referencias bibliográficas}

AlmeIDA, I. (1986). «Sémiotique et interpretation». Pré-publications, 15354. Urbino: Centro Internazionale di Semiotica e di Linguistica.

Alemán, J. y LABRERA, S. (1981). «Unaridad del significante». Boletín de Psicoanálisis, $\mathrm{n}^{\circ}{ }^{1}$. Madrid.

ARBoledA, R. (1987). «EI ALEC y algunas obras conexas». Glotta, v. 2, n. 3. Bogotá. 
ARgứllo, R. (1998). Ciudad Gótica, Esperpéntica y Mediática. Bogotá: Educativa Net.

Benveniste, E. (1966). Problemas de Lingüistica General. Traducción de Juan Almela. México: Siglo XXI.

Bernal, J. (1986). Antología de Lingüística Textual. Bogotá: Instituto Caro y Cuervo.

Castañares, W. (1987). «Ch. Peirce. Historia de una marginación». Revista de Occidente, n. ${ }^{\circ} 71$. Madrid.

Charaudeau, P. (1983). Langage et Discours. Eléments de sémiolinguistique. París: Hachette Université.

Dascal, M. (1978). La Semiología de Leibniz. Trad. de Luis Alfonso Palau. Universidad Nacional de Medellín, 1990.

DerridA, J. (1967). De la Gramatología. Trad. de Óscar del Barco. Argentina: Siglo XXI.

DOMíNGUEZ, E. (1990). «La crítica interdisciplinaria en la historiografía y el periodismo». Cuadernos del CIDI, n. ${ }^{\circ} 15$. Medellín: Universidad Pontificia Bolivariana.

DuCrot, O. (1986). Le dire et le dit. París: Minuit.

DUCROT, O. y TODOROV, T. (1972). Dicionario enciclopédico de las ciencias del lenguaje. Trad. de Enrique Pezzoni. México: Siglo XXI.

Eco, U. (1976). Tratado de Semiótica general. Trad. de Carlos Manzano. Barcelona: Lumen.

ElKanA, Y. (1977). La culture scientifique dans le monde contemporaine. Trad. de José Granés. Bogotá: Sociedad Colombiana de Epistemología.

Escamilla, J. (1987). «El Círculo de Análisis del Discurso». Glotta, vol. 2, Bogotá.

GARRONI, E. (1977). Ricognizione della Semiótica. Roma: Officina Edizioni. GeNETTE, G. (1972). Figures III. París: Seuil.

Góngora, A. (1991). Mapa del teatro en Santander (en mimeo). Bucaramanga: Universidad Industrial de Santander.

GreIMAS, A. J. (1966). Eléments pour une théorie de l'interprétation du récit mytique. París: Points.

- (1986). Sémiotique. Dictionare raisonné de la théorie du langage, T.2.

París: Hachette Université.

GoMBrich, E. (1959). Art and Ilusion. London: Phaidon Press.

GUMPERZ, J. (1974). «The Sociolinguistics of Interpersonal Communication».

n. ${ }^{\circ} 33$, Serie C. Urbino: Centro Internazionale di Semiotica e di Linguistica.

IRIARTE, G. (1991). Semiótica narrativa de la tradición oral (en Mimeo). Bogotá: Universidad Nacional.

JURADO, F. (1989). «Correlato religioso y roles actanciales en Talpa». Revista de Lingüistica y Literatura. Medellín: Universidad de Antioquia.

KRISTEVA, J. (1972). Semiótica. Trad. de José Martín Arancibia. Madrid: Fundamentos.

KuHN, T. (1962). «The Structure of Scientific Revolutions». Trad. en México: F.C.E. En Encyclopedia of Unified Science. University of Chicago. 
LACAN, J. (1966). Escritos. Trad. de Tomás Segovia. México: Siglo XXI.

LADRIERE, J. (1973).«Vie sociale et destinée». Cit. por Almeida (1986). Gembloux: Duculot.

LÉVI-Strauss, (1958). Antropología estructural. Trad. de Eliseo Verón. Buenos Aires: Eudeba.

- (1964). Lo crudo y lo cocido. Buenos Aires: Eudeba.

LlerenA, R. (1985). Memoria cultural en el vallenato. Medellín: Centro de Investigaciones Universidad de Antioquia.

LORENZANO, C. (1982). La estructura psicosocial del arte. Buenos Aires: Siglo XXI.

LOTMAN, J. (1977). «La cultura come mente colletiva e i problemi della intelligenza artificiale», n. ${ }^{\circ} 66$, Serie A. Urbino: Centro Internazionale di Semiotica e di Linguistica.

LYOTARD, J. F. (1984). La condición posmoderna. Madrid: Cátedra.

MARTIN-BARBero, J. (1987). De los medios a las mediaciones. México: Gustavo Gili.

Martinet, A. (1965). Elementos de Lingüística General. Madrid: Gredos.

MerTZ, E. (1985). Semiotic Mediation: Sociocultural and Psychological Perspectives. Florida: Academic Press, USA.

METZ, C. (1977). El significante imaginario. Trad. de Josep Elías. Barcelona: Gustavo Gili.

Montoya, J.; Moreno, J.G. y Xibille, J. «Teoría del Arte». Revista de Ciencias Humanas, n. ${ }^{\circ}$ 10. Medellín.

MonTOYA, J. (1991). «En torno a una semio-pragmática del discurso». Revista Universidad Nacional. Extensión cultural n. ${ }^{\circ} 29-30$. Medellín.

Moreno, J. G. (1991). «Peirce. Ponencia presentada en el Encuentro de Semiótica de la Universidad del Cauca». Popayán.

Paramo, G. (1989). «Lógica de los mitos». Revista Ideas y Valores, n. $^{\circ} 79$. Bogotá: Universidad Nacional.

Peirce, C. (1965). The Collectet Papers, C. Hartschorme, P. Weiss y A. Burks (eds.), vol. 8. Cambridge, Mass., U. S. A.

Pergolis, J. C. (1986). Sobre lo clásico en la arquitectura. Bogotá: Fondo Ed. Universidad Nacional.

RAMíreZ, A. (1986). «La lingüística del texto en la literatura». En Antología de la lingüística textual. Bogotá: Instituto Caro y Cuervo.

Restrepo, M. (1992). Ser signo interpretante. Bogotá: Significante de Papel.

REYes, L. y PARDo, G. (1991). Un modelo lingüístico para el análisis integral del discurso. Bogotá: Instituto Caro y Cuervo.

RicoeUR, P. (1971). «La communication». Actas du XV Congres de la Societé de Philosophie de Langue Française. Université de Montréal.

Rincón, A. (1992). Signo y lenguaje en San Agustín. Bogotá: Universidad Nacional.

RuBio, J. (1987). Explicar y comprender: Hermeneútica y Ciencias SocialesProblemas metodológicos (en mimeo). Bogotá. 
Sebeok, T. y Umiker, J. (1979). Sherlock Holmes y Charles S. Peirce: el método en la investigación. Barcelona: Paidós.

SERRANO, E. (1991). La manipulación enunciativa del saber en "Crónica de una muerte anunciada» (en mimeo). Cali.

Silva, A. (1986). Graffiti: una ciudad imaginada. Bogotá: Universidad Nacional.

- (1988). «La Semiótica y la Comunicación Social en Colombia». Revista Diálogos, n. ${ }^{\circ} 22$. Lima, Perú: Federación Latinoamericana de Facultades de Comunicación Social.

- (1993). «La Semiótica en América Latina: entre el ejercicio académico y su popularización». Revista de Ciencias de la Información, $\mathrm{n} .^{\circ} 8$. Madrid, España: Facultad de Ciencias de la Información, Universidad Complutense de Madrid.

TовóN, R. (1989). «Semiótica del silencio». Revista Coloquios Lingüísticos, n. ${ }^{\circ}$ 2. Medellín.

VAN DiJK, T. (1978). La ciencia del texto. Buenos Aires: Paidós.

VERÓN, E. (1977). «La semiosis social». Pre-publications, n. ${ }^{\circ}$ 64. Urbino: Centro Internazionale di Semiotica e di Linguistica. 